Ann. Sci. forest., 1982, 39 (1), 63-76.

\title{
Mesure des facteurs d'intensité de contrainte critiques et des taux de restitution d'énergie dans le bois sur éprouvettes entaillées
}

\author{
P. TRiboulot, P. JODIN, G. PLUVINAGE \\ Laboratoire de Fiabilité mécanique \\ Faculté des Sciences, Université de Metz \\ lle du Saulcy, F 57000 Metz.
}

\begin{abstract}
Résumé
A l'aide d'éprouvettes entaillées sollicitées en traction, nous avons testé une méthode de mesure de la complaisance de l'éprouvette permettant le calcul du taux de restitution d'énergie $G_{c}$. Les éprouvettes ont été prélevées dans le sens LT et TL dans trois essences différentes. Elles ont été sollicitées en mode I (ouverture des lèvres de l'entaille) et en mode mixte I + II (superposition d'une ouverture et d'un glissement droit des lèvres de l'entaille). Les résultats des essais ont montré que l'on pouvait déterminer $G_{I c}$ sans difficulté pour les éprouvettes prélevées dans le sens TL (mode I ou mode mixte I + II), mais qu'il fallait être prudent dans l'interprétation des résultats obtenus pour les éprouvettes prélevées dans le sens LT. On peut calculer ensuite aisément un facteur d'intensité de contrainte critique $\mathbf{K}_{\mathrm{I}}$ pour peu que l'on connaisse avec exactitude les valeurs des coefficients d'élasticité du matériau. Le facteur d'intensité de contrainte critique peut ensuite être utilisé pour dimensionner les constructions en bois.
\end{abstract}

\section{I. - Introduction}

Le bois est un des matériaux de construction les plus répandus et a été très largement utilisé depuis la préhistoire pour la construction des habitations, des outils, des armes ou des meubles.

De nos jours, son importance technologique est moindre, mais son incidence économique reste parfois plus importante que celle de certains métaux. Pour ces raisons, l'utilisateur et le concepteur de constructions en bois chercheront à mieux connaître les propriétés mécaniques du matériau afin d'améliorer les performances et la fiabilité de leurs réalisations, tout en cherchant à en réduire le coût.

Les principes régissant les constructions en bois sont, à l'heure actuelle encore très empiriques pour deux raisons :

- l'abondance relative du matériau et son faible prix de revient ont fait que l'on n'a pas hésité à surdimensionner les structures ; 
- l'extrême variabilité des propriétés mécaniques du matériau a également incité à surdimensionner plutôt qu'à chercher à éliminer systématiquement les causes de la variabilité.

Dans le but d'une meilleure connaissance du matériau, nous avons cherché à caractériser le bois vis-à-vis de sa résistance à la propagation brutale d'une fissure. C'est le mode par ouverture des lèvres de la fissure (mode I) qui retient le plus notre attention. Toutefois, un mode mixte par ouverture et glissement droit (mode II) a été aussi étudié (figure 1).
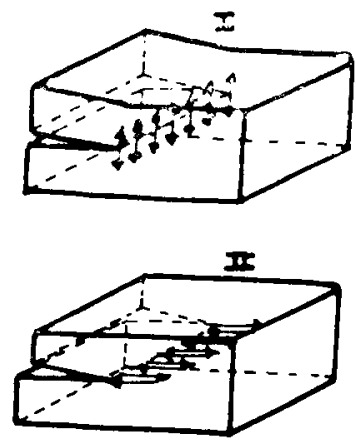

FIG. 1

Les deux modes de rupture I et II

The two modes of fracture

Après un rappel des principes de la mécanique de la rupture, nous présentons une étude bibliographique de son application au bois.

En conclusion, on montrera que la résistance à la propagation d'une fissure dans le bois peut être mesurée à l'aide d'une méthode simple et fiable et que, dans certains cas, on pourra dimensionner les constructions en bois à l'aide $d u$ facteur d'intensité de contrainte $\mathbf{K}_{\mathbf{I c}}$.

\section{II. - Eléments de la mécanique de la rupture des milieux anisothropes}

On fait, au départ, les hypothèses suivantes :

- homogénéité du matériau (acceptable dans la mesure où la dimension des éprouvettes est grande par rapport à la différence de diamètre entre deux cernes consécutifs) ;

- matrice de rigidité orthotrope ( 9 constantes); 
- comportement élastique dans les domaines de charges et de vitesse de sollicitations envisagés.

A partir de ces hypothèses, on peut envisager deux approches différentes du problème de la propagation d'une fissure : soit l'approche locale qui considère la distribution des contraintes au fond de la fissure (facteur d'intensité de contrainte $\mathrm{K}$ ), soit l'approche globale qui résulte d'un bilan énergétique relatif à la création de deux nouvelles surfaces de rupture (taux de restitution d'énergie G).

\section{Facteur d'intensité de contrainte $K$}

La figure 2 définit un système de coordonnées en fond de fissure ainsi que les contraintes associées à un petit élément de volume. Dans ce système, les contraintes en fond de fissure sont déterminées à l'aide des relations suivantes :

$$
\begin{aligned}
& \sigma_{x}=\frac{K_{I}}{\sqrt{2 \pi r}} f_{x}(\theta)+\frac{K_{I I}}{\sqrt{2 \pi r}} g_{x}(\theta) \\
& \sigma_{y}=\frac{K_{I}}{\sqrt{2 \pi r}} f_{y}(\theta)+\frac{K_{I I}}{\sqrt{2 \pi r}} g_{y}(\theta) \\
& \tau_{x y}=\frac{K_{I}}{\sqrt{2 \pi r}} f_{x y}(\theta)+\frac{K_{I I}}{\sqrt{2 \pi r}} g_{x y}(\theta)
\end{aligned}
$$

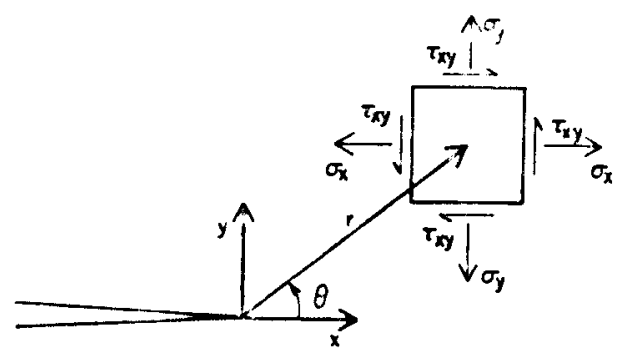

FIG. 2

Système de coordonnées et contraintes en fond de fissure

Coordinates and stresses system a crack tip

Ces relations permettent de déterminer les facteurs d'intensité de contrainte $\mathbf{K}_{\mathbf{I}}$ en mode $I$ et $K_{I I}$ en mode II si l'on dispose d'un moyen d'évaluer soit les contraintes autour du fond d'entaille, par mesure directe, soit les déplacements par le calcul (méthode des éléments finis par exemple). Les fonctions $f$ et $g$ sont établies à partir de la théorie de l'élasticité [méthode de WestergaARd (CorTen, 1972 ; SiH, 1965)]. Il y a rupture lorsque, par suite de l'augmentation des contraintes appliquées 
au corps fissuré, le facteur d'intensité de contrainte $K_{I}$ (ou $K_{I I}$ ) au fond de la fissure atteint une valeur critique $K_{I c}$ (ou $K_{\text {II }}$ ) caractéristique du matériau. Ce critère est largement utilisé dans les milieux isotropes élastiques où la répartition des contraintes est bien connue. Celle-ci étant plus difficile à déterminer dans les milieux orthotropes, l'utilisation du critère $\mathrm{K}$ est délicate.

\section{Taux de restitution d'énergie $G$}

$G$ exprime le taux de variation d'énergie correspondant à un petit accroissement de fissure $\delta a$. Sous une forme analytique, on écrit :

$$
\mathrm{G}=\frac{\mathrm{P}^{2}}{2 \mathrm{~B}} \frac{\partial \mathrm{C}}{\partial \mathrm{a}}
$$

où $\mathrm{P}$ est la charge appliquée, $\mathrm{B}$ l'épaisseur de l'échantillon, $\mathrm{C}$ la complaisance de l'éprouvette fissurée et (a) la longueur de la fissure. Lorsque $G$ atteint une valeur critique $G_{c}$, la rupture se produit. Ce critère a l'avantage de ne pas nécessiter la connaissance de la répartition des contraintes en fond de fissure. C'est ce qui le fait préférer au critère $\mathbf{K}$ dans le cas des matériaux orthotropes.

\section{Relation entre $G$ et $K$}

La formule utilisée pratiquement pour dimensionner les constructions est :

$$
\mathrm{K}_{\mathrm{Ic}}=\sigma^{\infty} \vee \pi \mathrm{a} . \mathbf{f} \text { (géométrie) }
$$

où $\sigma^{\infty}$ est la contrainte appliquée à la structure loin du défaut, (a) la dimension du défaut et $\mathrm{f}$ (géométrie) une expression qui dépend de la géométrie du corps fissuré et de ses propriétés élastiques.

$\mathrm{K}_{\mathrm{Ic}}$ ou $\mathrm{K}_{\text {IIe }}$ sont obtenus à l'aide des relations suivantes (SiH et al., 1965) :

$$
\begin{aligned}
& \mathrm{G}_{\mathrm{Ic}}=\mathrm{K}^{2} \mathrm{Ic}\left(\frac{\mathrm{S}_{11} \mathrm{~S}_{22}}{2}\right)^{1 / 2}\left[\left(\frac{\mathrm{S}_{2,2}}{\mathrm{~S}_{11}}\right)^{1 / 2}+\frac{2 \mathrm{~S}_{1 \mathrm{I}}+\mathrm{S}_{66}}{2 \mathrm{~S}_{11}}\right]^{1 / 2}=\mathrm{K}^{12} \times \mathrm{f}_{\mathrm{I}}\left(\mathrm{S}_{\mathrm{ij}}\right) \\
& \mathrm{G}_{1 \mathrm{Ic}}=\mathrm{K}^{2}{ }_{\mathrm{IIc}} \frac{\mathrm{S}_{11}}{\sqrt{2}}\left[\left(\frac{\mathrm{S}_{22}}{\mathrm{~S}_{11}}\right)^{1 / 2}+\frac{2 \mathrm{~S}_{12}+\mathrm{S}_{66}}{2 \mathrm{~S}_{11}}\right]^{1 / 2}=\mathrm{K}^{2}{ }_{\mathrm{IIc}} \times \mathrm{f}_{1 \mathrm{I}}\left(\mathrm{S}_{\mathrm{ij}}\right)
\end{aligned}
$$

où $\varepsilon_{1}=S_{i j} \sigma_{j} \quad \varepsilon_{i}=$ déformation, $\sigma_{j}=$ contrainte, $\mathrm{i}, \mathrm{j}=1,2,6$

avec $\varepsilon_{x}=\varepsilon_{1}, \varepsilon_{y}=\varepsilon_{2}$, les directions $x$, y étant celles de la figure $2 \varepsilon_{x y}=\varepsilon_{6}$

Connaissant la contrainte appliquée, on peut déterminer la dimension du défaut a.

Un rapport récent de l'un de nous (TRIBoulot, 1979) donne une liste à peu près complète des articles publiés sur la mécanique de la rupture appliquée au bois. La plupart des études portent sur le mode I sens TL. Quelques études existent en mode II et en mode mixte I + II. Il existe très peu d'études en mode I sens LT, en raison 
des difficultés dinterprétation dues au fait que la fissure se propage perpendiculairement à son plan.

\section{Mode I}

Le taux de restitution d'énergie du pin blanc a été mesuré par différents auteurs (DE BAISE et al., 1966; PoRTER, 1964) qui donnent des valeurs comprises entre 229 et $315 \mathrm{~J} / \mathrm{m}^{2}$. Schniewind \& Pozniak (1971), à l'aide d'une formule empruntée à la mécanique des miljeux isotropes donnent des valeurs de $K_{I}$. du douglas comprises entre 0,285 et $0,389 \mathrm{MNm}^{-3 / 2}$.

Johnson (1973) a mesuré le $\mathrm{K}_{\mathrm{I} \text { c }}$ de plusieurs essences réparties en trois groupes. I1 trouve, en moyenne, $0,330 \mathrm{MNm}^{-3 / 2}$ pour les résineux, $0,490 \mathrm{MNm}^{-3 / 2}$ pour les feuillus homogènes et $0,440 \mathrm{MNm}^{-3 / 2}$ pour les feuillus hétérogènes.

MURPHY (1978) a cherché à expliciter la valeur apparente du facteur d'intensité de contrainte $\mathrm{K}_{\mathrm{I}}$, mesuré dans le sens LR sur du douglas. Il montre que c'est une combinaison linéaire résultant d'une superposition d'une ouverture (mode $I, K_{I}$ ) et d'un glissement droit (mode II, $\mathrm{K}_{\mathrm{II}}$ ).

\section{Mode II}

La mesure a été faite sur une poutre sollicitée en flexion entaillée dans le plan de la fibre neutre (BARRETT \& Foschi, 1977) et confirme que le facteur d'intensité de contrainte en mode II $\mathrm{K}_{\mathrm{IIc}}$ est supérieur, en règle générale, à $\mathrm{K}_{\mathrm{Ic}}$.

\section{Mode mixte $I+I I$}

A l'aide d'éprouvettes à entaille latérale inclinée, Willıams \& BirCH (1976) ont tenté de déterminer une relation entre $K_{I}$ et $K_{I I}$ constituant un critère de rupture. La méthode utilisée est celle de la complaisance.

En conclusion de cet aperçu bibliographique, on peut remarquer :

— qu'il est très difficile d'établir une comparaison entre les différentes valeurs publiées, car les matériaux et les conditions d'expérimentation sont toujours différents. Il est seulement possible de comparer les ordres de grandeur ;

- que la validité des formules établies pour les matériaux isotropes est douteuse pour le bois dans le sens TL ou RL et elles sont totalement inapplicables dans le sens LT ou LR ;

- que ce n'est que très récemment que la mécanique de la rupture est appliquée au bois. De nombreuses expériences sont donc nécessaires pour tester la validité des théories existantes. 


\section{III. - Matériel et méthode}

Trois essences [le Douglas (pseudotsuga menziesii), le Hêtre (fagus sylvatica) et le Sipo (entandrophragma utile)] ont été sélectionnées.

Du point de vue de la mécanique de la rupture, on désigne l'orientation du prélèvement de l'échantillon avec deux lettres, la première désignant la normale au plan de l'entaille, la deuxième la normale au front de fissure dans le plan de l'entaille (figure 3). Par exemple une éprouvette prélevée dans le sens LT indique que le plan de l'entaille mécanique est perpendiculaire à l'axe longitudinal et que la normale du front de fissure est la direction tangente aux cernes annuels.

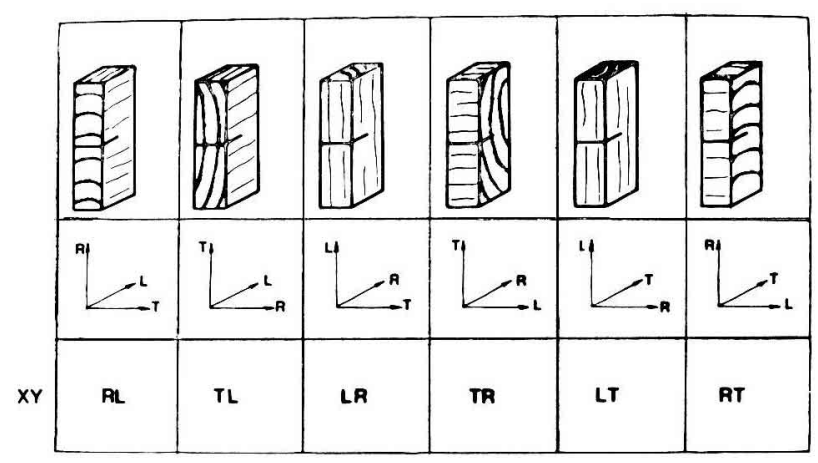

FIG. 3

Notation et désignation des sens de prélèvement Sample orientation

Nous avons procédé à des essais en mode I sens TL et sens LT et en mode mixte I + II sur des échantillons dont l'humidité était comprise entre 8 p. 100 et 12 p. 100. Parmi les nombreux types d'éprouvettes permettant de solliciter une fissure en mode I, nous avons retenu le type à entaille latérale en raison de sa facilité d'usinage et de mise en ouvre (figure 4a).

Les expériences réalisées en mode mixte I + II sur le Sipo sont identiques à celles réalisées en mode I. Les éprouvettes diffèrent au niveau de l'entaille qui est inclinée par rapport à la normale à l'axe de sollicitation, mais qui est maintenue dans le fil du bois (figure 4b). Les dimensions des éprouvettes ont été choisies de telle sorte qu'on puisse faire varier aisément le rapport a/W et que l'influence du dispositif d'attache soit négligeable. L'entaille est exécutée avec une scie et est prolongée par un trait de scie fine (ép. : $0,2 \mathrm{~mm}$ ) puis par un coup de lame de rasoir. Dans une série de pré-essais, nous avons fait varier l'épaisseur de l'échantillon entre 5 et $20 \mathrm{~mm}$ et nous avons constaté que celle-ci n'avait pas d'influence sur la mesure de G. Le montage expérimental est montré sur la photo 1. L'ouverture des lèvres de l'entaille est enregistrée à l'aide d'un extensomètre à lames relié à un enregistreur. Le fond de l'entaille est observé durant l'essai avec une lunette microscopique. L'ensemble est monté 
sur une machine de traction à traverse mobile de $100 \mathrm{kN}$ de capacité. Des essais à différentes vitesses de traverse ont montré que celle-ci avait une importance considérable. Nous avons par la suite fixé la vitesse des essais à $1 \mathrm{~mm} / \mathrm{mn}$, conformément à la norme ASTM D 143-52 section 55 .

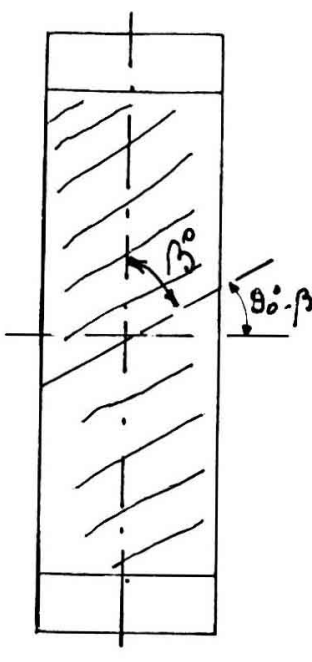

b

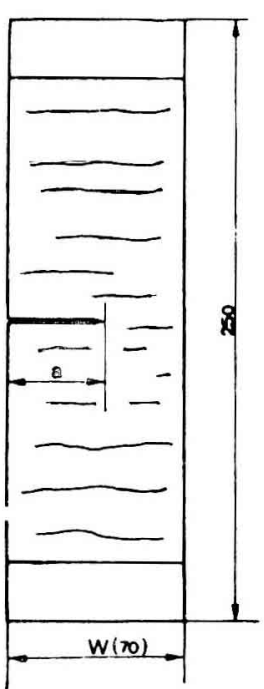

a

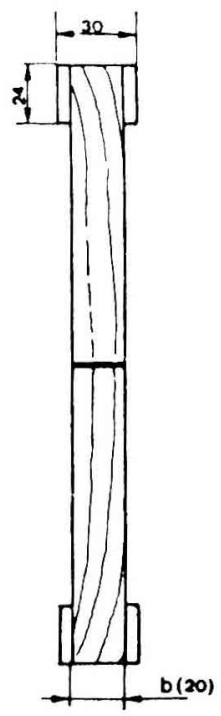

FIG. 4

Schéma des éprouvettes

Specimen design

La mesure de $G$ nécessite la connaissance de la charge $P$ et de la variation de la complaisance $\partial \mathrm{C} / \partial \mathrm{a}$. On enregistre donc des diagrammes charge appliquée ouverture des lèvres de l'entaille (figure 5). Sur ces diagrammes on peut déterminer la charge critique $P_{c}$ ainsi qu'il sera montré ci-dessous et la complaisance de l'éprouvette $\mathrm{C}=\frac{\text { ouverture }}{\text { charge }}=\frac{\delta}{\mathrm{P}}$ dans la partie linéaire. On répète cette expérience pour divers rapports $a / W$. On peut alors tracer un diagramme $C=f(a / W)$ (figure 6 ). On lisse les points expérimentaux avec une courbe de type exponentielle, on en déduit la valeur de $\partial \mathrm{C} / \partial \mathrm{a}$ pour chaque essai et on peut alors déterminer une valeur de $G_{C}$ pour chaque essai. La charge critique $P_{c}$ est définie comme étant le point où la linéarité de la courbe disparaît. Cette définition s'appuie sur le fait que, grâce à l'observation microscopique, on voit apparaître une pluie de sciure dans le fond de l'entaille, ainsi que dans certains cas des microfissures à la surface de l'éprouvette autour de l'entaille mécanique au moment où la linéarité disparaît. La charge peut être encore augmentée mais le comportement du matériau n'est plus linéaire ri réversible, ce qui indique qu'il y a eu propagation. La direction de propagation de la fissure est toujours celle du fil du bois. En conséquence, la fissure 
se propage dans le plan de l'entaille lorsque l'éprouvette est prélevée dans le sens TL, et dans une direction perpendiculaire au plan de l'entaille lors d'un prélèvement $\mathrm{LT}$.

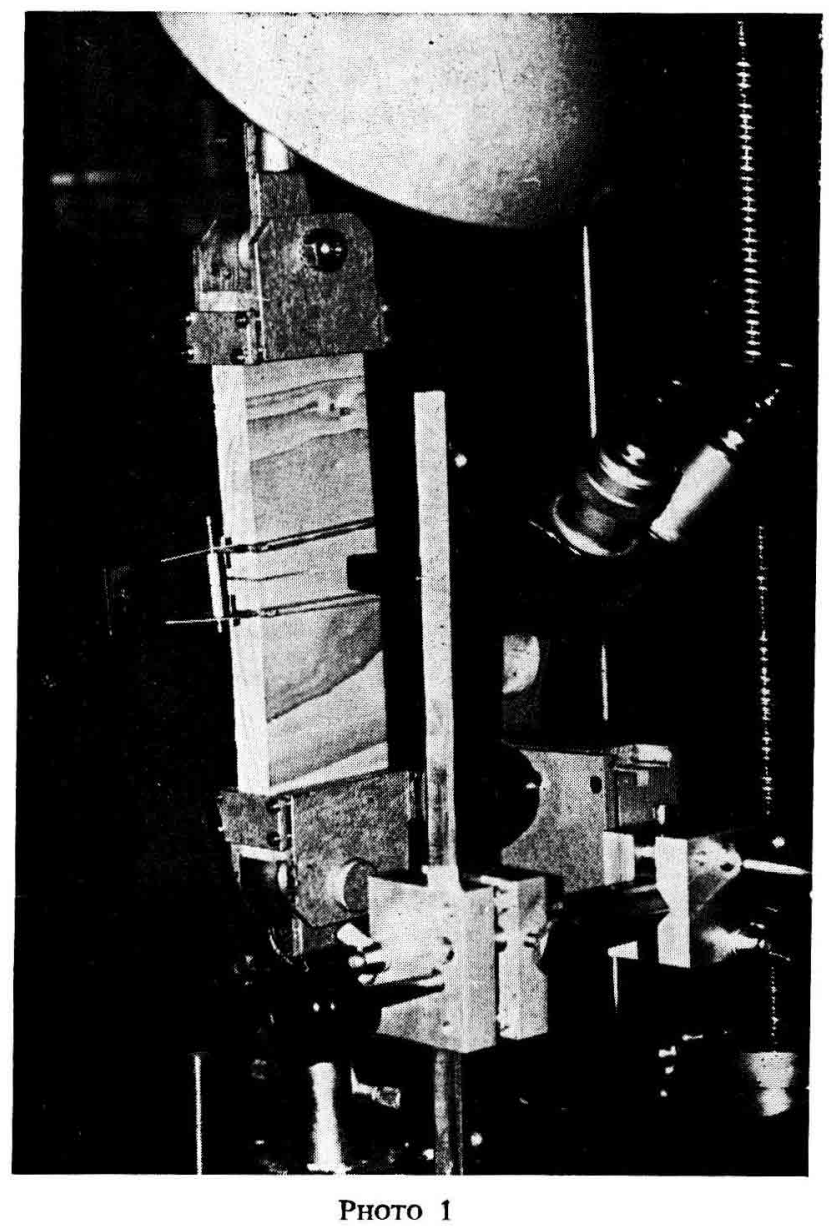

Montage expérimental

Experimental device

Mode I sens TL

Les résultats des expériences sont reportés dans le tableau 1. On y trouve l'expression analytique de la fonction de complaisance lissée à l'aide d'une méthode de moindres carrés, $r$ coefficient de corrélation correspondant à ce lissage, $G_{I \text { I: }}$ valeur moyenne du taux de restitution d'énergie calculé suivant la formule (2), $\Sigma$ l'écart type correspondant, $f_{I}\left(S_{i j}\right)$ un paramètre calculé selon la formule (4), $\bar{K}_{I \text { c la valeur }}$ moyenne du facteur d'intensité de contrainte critique calculée à l'aide de la for̀mule (4). 


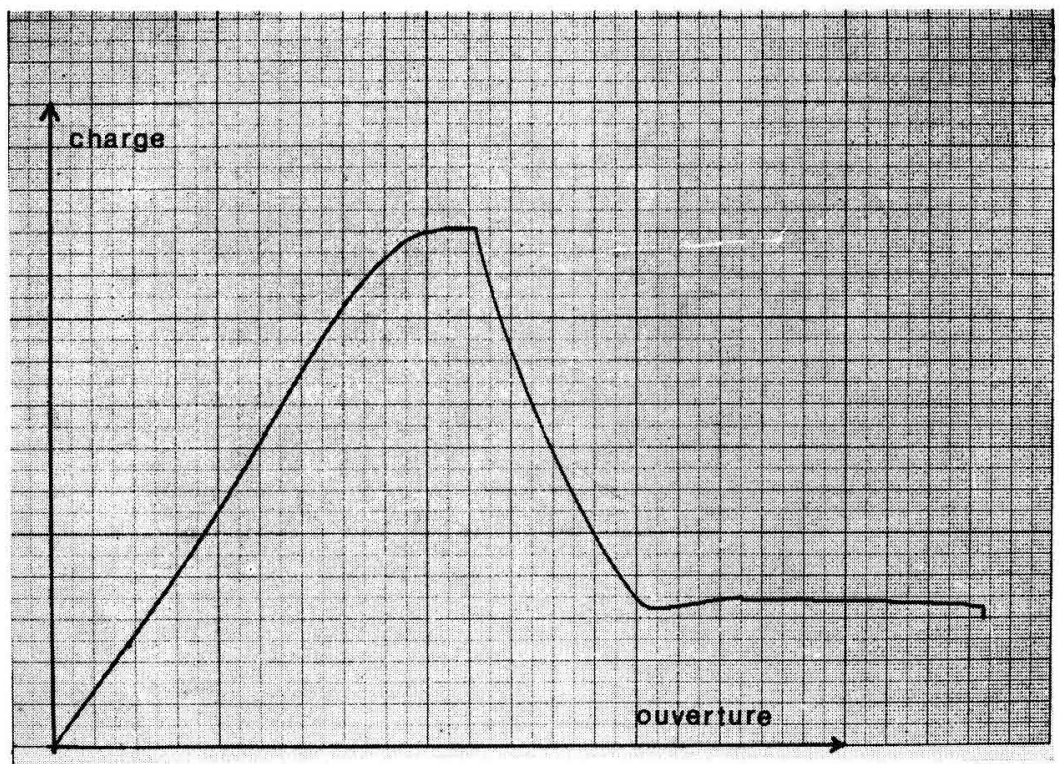

FIG. 5

Diagramme charge-ouverture

Typical load opening recording

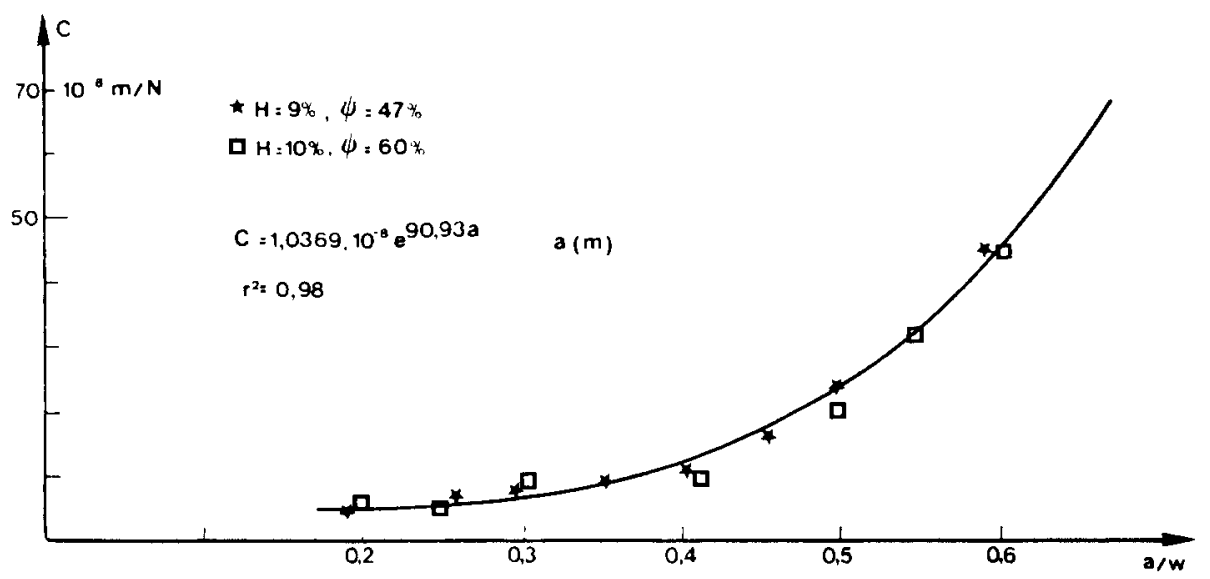

FIG. 6

Courbe de complaisance

Compliance curve 


\begin{tabular}{|c|c|c|c|c|c|c|c|c|c|c|}
\hline \multirow{9}{*}{ 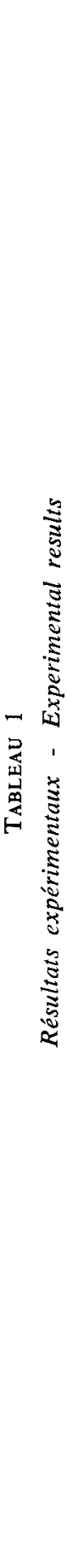 } & \multirow{3}{*}{$\begin{array}{l}\vec{F} \\
\ddot{D} \\
\ddot{\nu}\end{array}$} & 总 & $\infty$ & $\begin{array}{l}\overrightarrow{10} \\
10 \\
0 \\
0 \\
0 \\
2 \\
0 \\
0\end{array}$ & $\begin{array}{l}2 \\
0 \\
0\end{array}$ & $\frac{O}{7}$ & $\hat{\varrho}$ & $\begin{array}{l}n \\
n \\
6\end{array}$ & $\frac{\infty}{\stackrel{\infty}{0}}$ & $\begin{array}{l}8 \\
0\end{array}$ \\
\hline & & $\begin{array}{l}\frac{a}{0} \\
\frac{0}{00} \\
\overline{0}\end{array}$ & 尺्र & 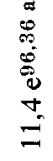 & $\begin{array}{l}\mathscr{2} \\
0 \\
0\end{array}$ & $\bar{m}$ & $\stackrel{m}{\infty}$ & $\hat{n}$ & $\begin{array}{l}0 \\
0 \\
0\end{array}$ & $\begin{array}{l}\infty \\
0 \\
0\end{array}$ \\
\hline & & 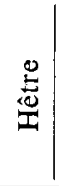 & $\leadsto$ & 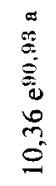 & 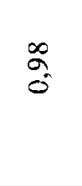 & $\vec{g}$ & $\widehat{\cong}$ & $\begin{array}{l}\infty \\
0 \\
n^{n}\end{array}$ & $\bar{\sigma}$ & $\stackrel{\sim}{\stackrel{0}{0}}$ \\
\hline & \multirow{3}{*}{ 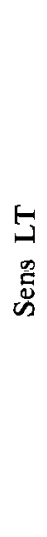 } & $\stackrel{\AA}{\circ}$ & $\stackrel{\infty}{-}$ & $\begin{array}{l}\sigma \\
0 \\
0 \\
0 \\
0 \\
a \\
j\end{array}$ & $\stackrel{\alpha}{\sigma}$ & $\begin{array}{l}8 \\
8 \\
\forall\end{array}$ & $\underset{8}{\mathscr{8}}$ & $\stackrel{0}{+}$ & $\begin{array}{l}\overrightarrow{0} \\
\text { in }\end{array}$ & ?े \\
\hline & & $\begin{array}{l}\frac{g}{d 0} \\
\frac{\partial}{\partial} \\
\stackrel{0}{0}\end{array}$ & $\stackrel{\infty}{\sim}$ & 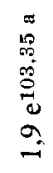 & $\begin{array}{l}\text { aे } \\
\text { ó }\end{array}$ & $\begin{array}{l}\mathscr{D} \\
8 \\
\dot{\nabla}\end{array}$ & $\frac{\infty}{6}$ & $\underset{-}{\mathbb{J}}$ & $\begin{array}{l}\circ \\
\stackrel{2}{+}\end{array}$ & $\stackrel{F}{0}$ \\
\hline & & 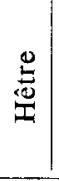 & $\bar{\sim}$ & 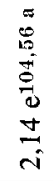 & $\stackrel{\infty}{2}$ & $\begin{array}{l}\stackrel{\infty}{7} \\
\underset{\gamma}{0}\end{array}$ & $\begin{array}{l}\tilde{n} \\
\infty \\
-\end{array}$ & $\underline{b}$ & $\underset{0}{\delta}$ & $\begin{array}{l}n \\
\infty \\
0 \\
0\end{array}$ \\
\hline & \multicolumn{2}{|c|}{$\stackrel{\mathscr{U}}{\stackrel{\mathscr{U}}{\Xi}}$} & & $\begin{array}{l}8 \\
\stackrel{8}{ } \\
x \\
z \\
\text { z }\end{array}$ & & $\stackrel{\Xi}{\Xi}$ & $\stackrel{\Xi}{\Xi}$ & $\begin{array}{l}\stackrel{\ominus}{0} \\
\times \\
\times \\
z \\
\stackrel{\partial}{\Xi}\end{array}$ & 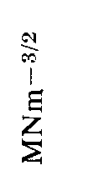 & $\begin{array}{l}\frac{N}{\infty} \\
1 \\
z \\
z \\
z\end{array}$ \\
\hline & \multicolumn{2}{|c|}{$\begin{array}{l}\frac{0}{\circ} \\
\text { है } \\
\text { ڤे }\end{array}$} & & 0 & $w$ & 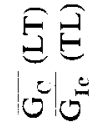 & $\omega$ & $\begin{array}{l}\overrightarrow{6} \\
\overrightarrow{6}\end{array}$ & 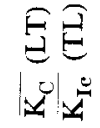 & W \\
\hline & & & 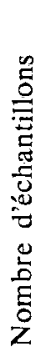 & 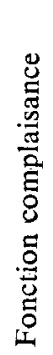 & 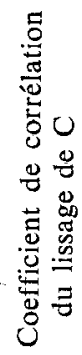 & 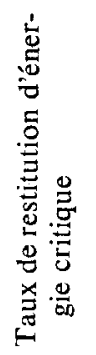 & 总 & 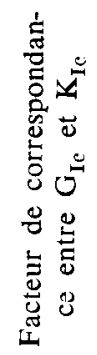 & 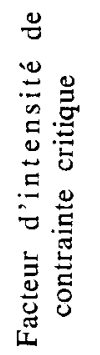 & 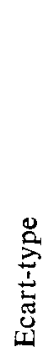 \\
\hline
\end{tabular}


A titre de comparaison, un alliage d'aluminium type 2618 A a un taux de restitution d'énergie critique $G_{I c}$ de $5555 \mathrm{~J} / \mathrm{m}^{2}$ environ. Le bois sollicité en mode I sens TL est donc relativement fragile. D'autre part, on montre que les valeurs de $G_{I c}$ obtenues sont, dans les limites de la dispersion, indépendantes $\mathrm{du}$ rapport $\mathrm{a} / \mathrm{W}$. De plus, les observations microscopiques au fond de l'entaille montrent bien qu'il y a naissance d'une microfissure au moment où la linéarité de la courbe charge - ouverture disparaît. On a montré également qu'à partir de ce point, le comportement du matériau n'était plus linéaire ni réversible. Il y a donc eu propagation de l'entaille dans son plan. Ces considérations permettent d'estimer que les conditions de validité de la mécanique linéaire élastique de la rupture sont remplies et que notre détermination de $\mathrm{G}_{\mathrm{Ic}}$ est une mesure réelle de la résistance à la propagation brutale d'une fissure.

On retiendra deux sources d'incertitude principales sur la valeur de $\mathbf{K}_{\mathbf{I c}}$ calculée. La première est la variabilité naturelle du matériau. Nous avons cherché à la réduire le plus possible en utilisant des échantillons soigneusement sélectionnés et en effectuant une statistique sur une population d'échantillons provenant du même arbre. Une autre source est introduite lors de l'évaluation du paramètre $f\left(S_{i j}\right)$. Nous avons utilisé pour ce calcul des données déjà anciennes (KollmanN, 1968).

\section{Mode I sens $L T$}

Dans le cas d'une expérience en mode I, des considérations théoriques (ERdogan \& SiH, 1963) prévoient que la fissure se propagera dans le plan de l'entaille. Or, dans toutes les expériences dans le sens LT, la fissure s'est propagée perpendiculairement à son plan. L'hypothèse du mode I n'est plus valable. C'est la raison pour laquelle on a noté dans le tableau 1 le taux de restitution d'énergie et le facteur d'intensité de contrainte sans lindice $I$. On note que les valeurs de $G_{c}$ dans ce cas sont environ dix fois supérieures à celles obtenues dans le sens TL.

La propagation dans la direction perpendiculaire au plan de l'entaille doit pouvoir s'expliquer à l'aide d'un critère de contrainte locale. On sait en effet que les résistances mécaniques dans le sens longitudinal et dans le sens tangentiel sont très différentes. SiH (1965) a donné des expressions des contraintes en fond de fissure pour un matériau orthotrope. On peut calculer ces contraintes dans quelques directions et vérifier que la fissure va se propager dans la direction de plus faible résistance.

\section{Mode mixte $I+I I$}

La figure 7 montre l'influence de l'angle $\beta$ que font l'entaille et le fil $\mathrm{du}$ bois avec l'axe de chargement. On observe une variation brutale de $K_{\text {Ic }}$ aux environs de $65^{\prime \prime}$. Les valeurs obtenues pour les angles inférieurs à $60^{\prime \prime}$ sont de l'ordre de grandeur de celle obtenue pour le mode I pur sens TL.

Le brusque changement du comportement du bois aux environs de $65^{\circ}$ suggère un changement de mécanisme gouvernant la rupture.

On suppose que dans la partie de la courbe (figure 7) comprise entre 0 et $60^{\circ}$, c'est le mode I qui gouverne la rupture et que dans la partie comprise entre $60^{\circ}$ 
et $90^{\circ}$, c'est le mode II qui domine. Williams \& Birch (1976) ont établi la relation $\mathrm{K}_{\mathrm{IIc}} / \mathrm{K}_{\mathrm{Ic}}=\left(\mathrm{E}_{\mathrm{L}} / \mathrm{E}_{\mathrm{T}}\right)^{1 / 4}$. Dans la configuration de notre éprouvette, on montre que $\mathrm{K}_{\mathrm{II}} / \mathrm{K}_{\mathrm{I}}=\operatorname{tg} \beta$.

Si on admet qu'au point de discontinuité les mécanismes par mode $I$ et par mode II sont simultanément vérifiés, on doit avoir $K_{I I c} / K_{I c}=\operatorname{tg} \beta=\left(E_{I_{0}} / E_{T}\right)^{1 / 4}$. Soit dans le cas du Sipo $\beta \sim 65^{\circ}$, ce qui correspond aux observations. Ceci est en accord avec les mesures de module d'élasticité en fonction de l'angle des fibres (Kollman, 1968 ; LeE, 1965).

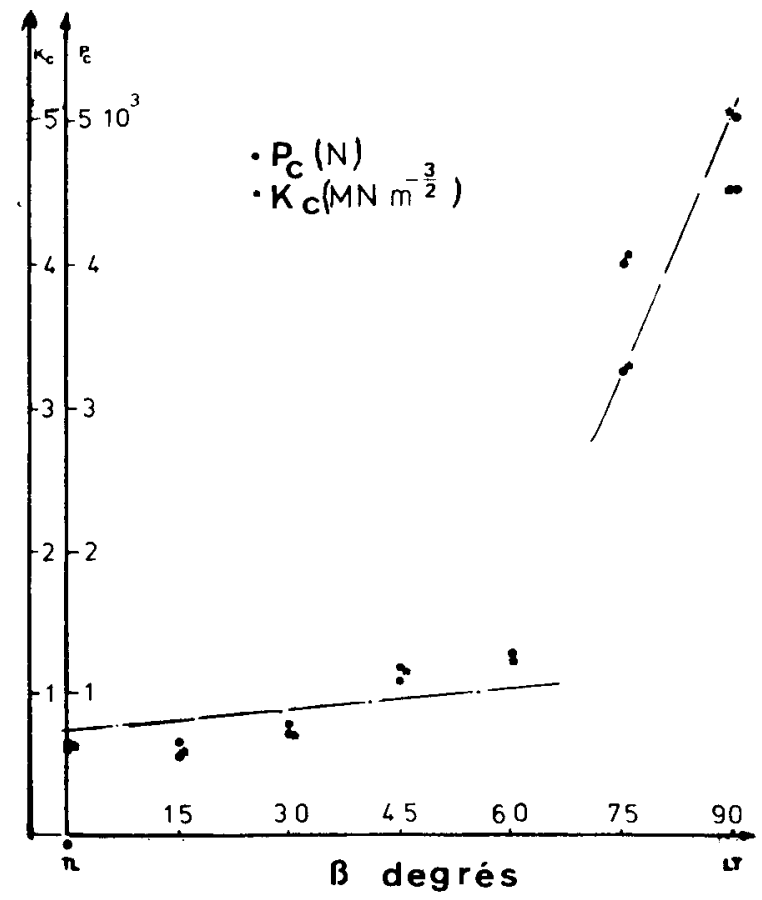

FIG. 7

Influence de l'angle de l'entaille et $d u$ fil du bois avec l'axe de chargement Influence of angle $\beta$ on critical load and stress intensity factor

\section{IV. - Conclusion}

On a donc montré dans cette étude que le facteur d'intensité de contrainte critique et le taux de restitution d'énergie critique du bois pouvaient être mesurés de façon fiable.

Dans le sens LT on calcule en fait un facteur d'intensité de contrainte apparent qui peut correspondre à la superposition de plusieurs mécanismes de rupture. L'analyse 
des phénomènes étudiés, en liaison avec la structure du bois, doit permettre de comprendre le mécanisme de rupture.

Dans le sens TL en mode I comme en mode mixte, on pourra utiliser les valeurs de $K_{\text {I. }}$ pour dimensionner les constructions. Il faut en effet être bien conscient que le bois est un matériau comportant des défauts naturels de dimensions non négligeables par rapport à celles des sciages utilisés en construction. De plus, par usinage et assemblage, on introduit des défauts artificiels qui peuvent être à la source de ruptures catastrophiques. Les considérations théoriques développées dans notre travail visent essentiellement à s'assurer que la grandeur mesurée correspond bien au comportement réel du matériau et que cette valeur peut être utilisée pour anéliorer la fiabilité des constructions en bois.

$$
\text { Reģu pour publication en avril } 1981 .
$$

\section{Remerciements}

Ce travail a pu être mené à bien grâce à l'aide de la D.G.R.S.T. Nous remercions très vivement la Station de Recherches sur la Qualité des Bois de l'I.N.R.A. à Champenoux, ainsi que le Centre Technique Forestier Tropical à Nogent-sur-Marne, qui nous ont procuré des échantillons soigneusement sélectionnés.

\section{Summary \\ Critical stress intensity factors and strain energy release rate in notched wood specimens}

Critical strain energy release rate measurements were made on notched wood specimens, using the compliance method. The specimens were cut out of three species in the TL and LT directions. Mode I (crack opening) and mixed mode I + II (crack opening + shearing of crack lips) were studied. Results show that $G_{l \text {. }}$ can be determined without difficulties in the TL direction. In the LT direction, the interpretation of the results is more difficult. The values of $\mathrm{K}_{\mathrm{I} \text { c }}$ obtained from $\mathrm{G}_{\mathrm{I}}$ can be used for sizing wood constructions.

\section{Références bibliographiques}

BarRet .D., Foschi R.O., 1977. Mode Il stress intensity factors for cracked wood beams. Eng. Fracture Mcch., 9, 371-378.

De Baisl: G.R., Porter A.W., Pentoney R.E., 1966. Morphology and mechanics of wood fracture. Mater. Res. Stands, 6, 493-499.

Corten H.T., 1972. In : «Fraciure, vol. VII : Fracture of non-metals and composites» (H. Liebowitz Ed.), chap. 9, pp. 675-769, New York.

Erdogan F., Sih G.C., 1963. J. Basic Eng., 85 C, 519-527.

Guitard D., Seichepine A., 1979. Comportement élastique du bois naturel : considérations préliminaires, Rapport Instifut National Polytechnique de Lorraine.

Johnson J.A., 1973. Crack initiation in wood plates. Wood Sci., 6, 151-158. 
KnotT J.F., 1973. Fundamentals of fracture mechanics. London, Butterworths, p. 105.

Kollmann F.F.P., 1968. Principles of wood science and technology, tome 1, Solid Wood, Springerverlag Ed., New York Inc.

LEE I.D.G., 1965. Ultrasonic pulse velocity testing considered as a safety measure for timber structures. Second Symposium on the NDT of Wood Washington State University.

MURPhy J.F., 1978. Using fracture mechanics to predict failure of notched wood beams, First International Conference on Wood Fracture, BANFF, Canada.

PARIS P.C., SiH G.C., 1964. Stress analysis of cracks. In : Fracture Toughness Testing and its Applications, A.S.T.M. S.T.P. 381, 80-83.

Porter A.W., 1964. On the mechanics of fracture in wood. For. Prod. J., 14, 325-331.

Schniewind A.P., Pozniak R.A., 1971. On the fracture toughness of Douglas fir wood. Eng. Fracture Mech., 2, 223-233.

Sih G.C., Paris P.C., Irwin G.R., 1965. On cracks in rectilinearly anisotropic bodies. Int. J. Fracture, 1, 189-203.

Triboulot P., Jodin P., Pluvinage G., 1980. Rapport de contrat D.G.R.S.T. n 78.7.2028 (à paraître).

Williams J.G., BiRCH M.W., 1976. Mixed mode fracture in anisotropic media. In : Cracks and Fracture, A.S.T.M. S.T.P. 601, 125-137. 